\title{
Gene mutational pattern and expression level in 560 acute myeloid leukemia patients and their clinical relevance
}

\author{
Yong-Mei Zhu ${ }^{\dagger}$, Pan-Pan Wang ${ }^{\dagger}$, Jin-Yan Huang, Yun-Shuo Chen, Bing Chen, Yu-Jun Dai, Han Yan, Yi Hu, \\ Wen-Yan Cheng, Ting-Ting Ma, Sai-Juan Chen* and Yang Shen ${ }^{*}$
}

\begin{abstract}
Background: Cytogenetic aberrations and gene mutations have long been regarded as independent prognostic markers in AML, both of which can lead to misexpression of some key genes related to hematopoiesis. It is believed that the expression level of the key genes is associated with the treatment outcome of AML.

Methods: In this study, we analyzed the clinical features and molecular aberrations of 560 newly diagnosed non-M3 AML patients, including mutational status of CEBPA, NPM1, FLT3, C-KIT, NRAS, WT1, DNMT3A, MLL-PTD and IDH1/2, as well as expression levels of MECOM, ERG, GATA2, WT1, BAALC, MEIS1 and SPI1.

Results: Certain gene expression levels were associated with the cytogenetic aberration of the disease, especially for MECOM, MEIS1 and BAALC. FLT3, C-KIT and NRAS mutations contained conversed expression profile regarding MEIS1, WT1, GATA2 and BAALC expression, respectively. FLT3, DNMT3A, NPM1 and biallelic CEBPA represented the mutations associated with the prognosis of AML in our group. Higher MECOM and MEIS1 gene expression levels showed a significant impact on complete remission (CR) rate, disease free survival (DFS) and overall survival (OS) both in univariate and multivariate analysis, respectively; and an additive effect could be observed. By systematically integrating gene mutational status results and gene expression profile, we could establish a more refined system to precisely subdivide AML patients into distinct prognostic groups.
\end{abstract}

Conclusions: Gene expression abnormalities contained important biological and clinical informations, and could be integrated into current AML stratification system.

Keywords: Acute myeloid leukemia, Gene expression, Mutation, Prognosis

\section{Background}

Acute myeloid leukemia (AML) is a group of hematological malignancies, arising from stem cells, whose leukemogenesis and clinical behavior was deeply affected by the underlying cytogenetic and molecular abnormalities [13]. Classic cytogenetic aberrations such as chromosomal translocations to form oncogenic fusion genes via rearrangement of coding sequences of the involved partner genes, such as $\mathrm{t}(15 ; 17), \mathrm{t}(8 ; 21), \mathrm{t}(16 ; 16) / \mathrm{inv}(16)$, have long

\footnotetext{
*Correspondence: sjchen@stn.sh.cn; shen_yang@126.com

${ }^{\dagger}$ Yong-Mei Zhu and Pan-Pan Wang contributed equally to this work Department of Hematology, Shanghai Institute of Hematology, RuiJin Hospital Affiliated to Shanghai Jiao Tong University School of Medicine, 197 Ruilin Road II, Shanghai 200025, China
}

been considered as diagnostic markers of each subgroup of AML, and even served in the surveillance of minimal residual disease (MRD), and more importantly, designing tailored treatment for the disease [4-8]. For more refined stratification, or redefinition of AML, molecular analysis of gene mutation with potential clinical relevance has been more widely used in recent years $[9,10]$. Traditionally, it was suggested that genetic abnormalities in leukemia could be roughly grouped into two classes according to their roles in pathogenesis: Class I, mutations involving signal transduction pathways and giving rise to proliferative advantages to leukemia clones, exemplified as $C-K I T$, FLT3 and NRAS; and Class II, mutations affecting transcription factors (TF) or co-factors and causing impaired 
differentiation such as point mutation of CEBPA, $A M L 1$ and gene fusion of $A M L 1-E T O$ [11]. In our previous work, a Class III mutation associated with epigenetic modifier was proposed, such as DNMT3A, IDH1, IDH2, and TET2, which shared a common feature of aggressive diseases, old age and poor prognosis [12]. With the development of second generation sequencing technology, a greater number of new gene mutations were identified in AML; which provides opportunities to more comprehensively understand and overview the gene events in the disease from a panoramic angle. Through whole genome and exome sequencing in 200 AML patients, Ley et al. suggested 9 categories of gene mutations in AML: NPM1, activated signaling, myeloid TFs, TF fusions, DNA methylation, chromatin modifier, tumor suppressors, cohesin complex, and spliceosome. However, their function and potential clinical translation in guiding treatment and judging prognosis should be further confirmed in future clinical trials [13].

Until now, the roles of a sizable portion of gene mutations are addressed and integrated in clinical practice. For instance, FLT3 and CEBPA mutations being the most common gene mutations in Western and Chinese populations respectively; represent poor and favorable indicators in AML (biallelic for CEBPA) [11, 12, 14-16]. Gene mutations associated with epigenetic modification are also considered as poor factors, such as $M L L, D N M T 3 A$, TET2, and ASXL1 mutations [12, 17, 18], while mutant NPM1 is regarded as a favorable one [12, 19, 20]. In addition, numerous clinical studies have proven the role of tumor suppressor genes such as TP53 and WT1 in cytogenetic normal AML (CN-AML) [21-24] and C-KIT in core binding factor AML (CBF-AML) [25]. Metallothionein III (MT3) may also act as a tumor suppressor gene of which the promoter hypermethylation can inactivate the gene and downregulate its expression level in pediatric AML [26]. Similar to cytogenetic abnormalities, gene mutation events are now involved in the classification or nomenclature in AML. In a recent large series of 1540 patients, clinical relevance of gene mutations was analyzed, and a new genomic classification of acute myeloid leukemia was proposed, which includes the categories of mutated chromatin, RNA-splicing genes, TP53 mutations, biallelic CEBPA mutations, MLL fusion gene, GATA2, MECOM, IDH2, and $\mathrm{t}(6 ; 9)(\mathrm{p} 23 ; \mathrm{q} 34) / D E K-$ NUP214, with each of the subtypes presenting distinct clinical behaviors [27].

How these gene mutations are involved in the leukemogenesis needs further investigation. It is believed that normal hematopoiesis and cellular differentiation is highly dependent on the transcriptional regulation systems. The expression of lineage-determining transcription factor is in strict time order. Gene alterations, including fusions and mutations, could lead to the abnormal expression of key genes, and these kinds of misexpression disrupt the TF-dependent genetic network. In recent years, the expression level of several genes became research interest, exemplified as $M E C O M$ (also termed EVI1 and PRDM3), BAALC, ERG and WT1. A remote GATA2 hematopoietic enhancer alteration in inv(3) (q21;q26) by activating EVI1 expression was reported [28]. It is believed that the expression level of these genes is negatively associated with the treatment outcome of AML [29-33]; however, the results are controversial between western and eastern countries especially for $B A A L C$ and ERG [34,35]. The role of some new gene markers, such as MEIS1, which is up-regulated by $M L L$ abnormalities, still needs to be addressed in AML [36, 37].

Hence, we performed this study to systemically investigate the role of a series of gene expression in AML, including previously known ones and newly established ones. Moreover, we intent to integrate these new markers into current established gene mutation profile to provide a more precise stratification of AML.

\section{Patients and methods}

\section{Patients}

The newly diagnosed non-M3 patients were selected from Shanghai Institute of Hematology (SIH). Patients with leukemia either transformed from myelodysplasia syndrome (MDS) or secondary to other malignancies were excluded from this study. Cytogenetic analysis was performed centrally in SIH in every patient. The bone marrow (BM) samples of de novo AML patients were studied mostly by R- and/or G-banding analysis, and were confirmed in most cases with relevant molecular markers [38].

This study was approved by the ethic board of Ruijin hospital. All patients had given informed consent for both treatment and cryopreservation of BM and peripheral blood (PB) according to the Declaration of Helsinki.

\section{Treatment protocols}

Younger AML patients (age $\leq 60)$ received standard first line treatment of DA like regimen, which consisted of daunorubicin $45-60 \mathrm{mg} / \mathrm{m}^{2}, \mathrm{D} 1-3$; and Ara-C $100 \mathrm{mg} / \mathrm{m}^{2}$, D1-7. In the consolidation therapy, they were treated with high-dose cytarabine based chemotherapy for 4 cycles. For old patients (age $>60, n=86$ ), the treatment was mainly decided by the physician: fit patients underwent a regimen similar to younger patients, but with a reduced consolidation cycles of high dose Ara-C to 2 cycles; unfit patients, underwent either low dose treatment, demethylation treatment or palliative care [39]. 


\section{Molecular genetic analysis}

Gene mutations/fusions were detected as previously reported [12]. The WT1 [40] and ABL1 control gene [41] RQ-PCR assays were performed as described before. The MECOM, ERG, GATA2, BAALC, MEIS1 and SPI1 expression levels were quantified by using the TaqMan Gene Expression Assay assays, according to the manufacturer's instructions (Assay ID: Hs00602795_m1, Hs01554629_m1, Hs00231119_m1, Hs00227249_m1, Hs01017441_m1, Hs02786711_m1, respectively). These seven genes' transcripts were normalized to $A B L 1$ by using the respective plasmid standards to generate normalized copy numbers. Reactions were performed using ABI ViiA ${ }^{\mathrm{TM}} 7$ (Life technologies, USA). Each sample was analyzed in duplicate. Data were reported using a common threshold of 0.1. Positive and negative controls were included in all assays.

\section{Statistical analyses}

Fisher's exact $P$ test was used to compare the gene expression levels in different subgroups, as well as the difference of CR rates. One way Anova test was used to compare the clinical features such as age and WBC count in different groups. OS was measured from the date of disease diagnosis to death (failure) or alive at last follow-up (censored). DFS was defined as the duration from the documentation of CR to treatment failure such as relapse, refractory disease, death, or alive in CR at last follow-up (censored). Kaplan-Meier analysis was used to calculate the distribution of OS and DFS. Hazard ratio analysis was performed to compare the difference of survivals. Binary logistic regression and COX model was used for the multivariate analysis of associations between mutational status and the achievement of CR and OS and DFS, respectively. A limited backward selection procedure was used to exclude redundant variates. All above statistical procedures were performed with the SPSS statistical software package, version 16.0.

\section{Results}

\section{Gene expression level in AML}

Gene expression levels of MECOM, ERG, WT1, GATA2, BAALC, MEIS1 and SPI1 in bone marrow (BM) of de novo AML patients were shown in Additional file 1: Figure S1.

We cut the expression level of WT1, BAALC and $E R G$ into high and low group by their median values in patients according to the previous reports [30, 32]. For the continuance of analysis, the cut-off levels of $M E C O M$, MEIS1, GATA2 and SPI1 were also chosen at their median values. Survival analysis showed that median value as cut-off value could separate patients with different prognosis in MECOM, MEISI and SPI1 expression group (OS: $\mathrm{P}<0.001, \mathrm{P}<0.001$ and $\mathrm{P}=0.010$, respectively), while using cut-off value at quartile 1 (Q1) or Q3 would lose the power to separate the patients (Additional file 2: Figure S2).

\section{Patient characteristics and gene expression level}

560 patients were entered into this study including 474 young patients (age $\leq 60$ years old) and 86 elderly patients (age $>60$ years old). The patients were classified into 3 groups: Group 1, Core Binding Factor AML (CBF-AML), which includes 89 patients, Group 2, Cytogenetic intermediate risk AML, which includes 401 patients with normal karyotype (CN-AML, 320 patients) or cytogenetic aberration without prognostic significance, and Group 3, Cytogenetic high risk patients (55 patients) group. Among group 2, young patients with normal karyotype (265 patients) or insignificant cytogenetic aberration accounted for $84 \%$ (336 patients), while elderly patients accounted for 16\% (65 patients) including $55 \mathrm{CN}$-AML patients. Cytogenetic results were failed or unavailable in 15 patients. The distribution of the patients in $\mathrm{FAB}$ classification and cytogenetic abnormalities of enrolled patients was listed in Additional file 3: Table S1.

Age was strongly associated with several gene expression levels. The patients with higher MEISI expression had the higher median age $(\mathrm{P}=0.026)$. Similarly, in old patients (age $>60)$, more patients harbored higher MEIS1 and SPI1 expression levels $(66.3 \%$ vs. 33.7\%, $\mathrm{P}=0.001 ; 60.5 \%$ vs. $39.5 \%, \mathrm{P}=0.035$, respectively). Female patients tended to have higher expression of MECOM, MEIS1 and WT1 $(\mathrm{P}=0.038, \mathrm{P}=0.010$ and $\mathrm{P}=0.032$, respectively). The high SPI1 expression group manifested the feature of high WBC count at disease presenting $(\mathrm{P}=0.003)$. The detailed clinical features were shown in Table 1.

\section{Cytogenetic aberrations and gene expression level}

There was a difference of gene expression levels including those of MECOM, MEIS1, SPI1, ERG, WT1, GATA2, and $B A A L C$ in different cytogenetic risk groups. In CBFAML, MEIS1 and WT1 (both $\mathrm{P}<0.001$ ) were lower in CBF $\alpha$-AML $(\mathrm{t}(8 ; 21))$, but higher in CBF $\beta$-AML (inv(16)) $(\mathrm{P}=0.001$ and $<0.001$, respectively); while $B A A L C$ was higher in both groups (both $\mathrm{P}<0.001$ ). And SPI1 was high in CBF $\beta$-AML $(\mathrm{P}<0.001)$ as previously reported [42]. In CN-AML, MECOM and BAALC were lower (both $\mathrm{P}<0.001)$, while GATA2 was higher $(\mathrm{P}=0.014)$. Particularly, $M E C O M$ tended to have a higher level in poor risk factors both in $11 \mathrm{q} 23$ rearrangement $(\mathrm{P}=0.001)$ and others $(\mathrm{P}=0.015) .11 \mathrm{q} 23$ abnormalities were associated with higher expressions of MECOM $(\mathrm{P}=0.001)$, MEIS1 $(\mathrm{P}=0.003)$, SPI1 $(\mathrm{P}=0.031)$, WT1 $(\mathrm{P}=0.032)$, and interestingly, a lower $B A A L C$ expression $(\mathrm{P}=0.003)$ in our 
Table 1 Clinical characteristics and gene expression level

\begin{tabular}{|c|c|c|c|c|c|c|}
\hline \multirow[t]{2}{*}{ Gene expression } & \multicolumn{3}{|l|}{ Age (years) } & \multicolumn{2}{|c|}{ Gender, n (\%) } & \multirow{2}{*}{$\begin{array}{l}\text { WBC count, } \times 10^{9} / L \\
\text { Median (range) }\end{array}$} \\
\hline & Median (range) & $\leq 60, n(\%)$ & $>60, \mathrm{n}(\%)$ & Male & Female & \\
\hline \multicolumn{7}{|c|}{ MECOM (missing = 12) } \\
\hline $\operatorname{Low}(n=274)$ & $42.5(1-81)$ & $238(51.4)$ & $36(42.4)$ & $168(61.3)$ & $106(38.7)$ & $14.2(0.34-453.0)$ \\
\hline High $(n=274)$ & $43(1-83)$ & $225(48.6)$ & $49(57.6)$ & $144(52.6)$ & $130(47.4)$ & $13.9(0.50-376.8)$ \\
\hline P & 0.448 & & 0.125 & 0.038 & & 0.515 \\
\hline \multicolumn{7}{|l|}{ MEISI } \\
\hline $\operatorname{Low}(n=280)$ & $42(2-80)$ & $251(53.0)$ & $29(33.7)$ & $175(62.5)$ & $105(37.5)$ & $13.7(0.65-453.0)$ \\
\hline High $(n=280)$ & $45(1-83)$ & $223(47.0)$ & $57(66.3)$ & $145(51.8)$ & $135(48.2)$ & $15.3(0.34-389.4)$ \\
\hline P & 0.026 & & 0.001 & 0.010 & & 0.409 \\
\hline \multicolumn{7}{|l|}{ SPI1 } \\
\hline $\operatorname{Low}(n=280)$ & $42(1-81)$ & $246(51.9)$ & $34(39.5)$ & $165(58.9)$ & $115(41.1)$ & $10.6(0.5-453.0)$ \\
\hline $\operatorname{High}(n=280)$ & $44(1-83)$ & $228(48.1)$ & $52(60.5)$ & $155(55.4)$ & $125(44.6)$ & $16.9(0.34-298.5)$ \\
\hline P & 0.113 & & 0.035 & 0.393 & & 0.003 \\
\hline \multicolumn{7}{|l|}{$E R G$} \\
\hline $\operatorname{Low}(n=280)$ & $44(1-81)$ & $238(50.2)$ & $42(48.8)$ & $151(53.9)$ & $129(46.1)$ & $13.1(0.34-389.4)$ \\
\hline High $(n=280)$ & $41(1-83)$ & $236(49.8)$ & $44(51.2)$ & $169(60.4)$ & 111 (39.6) & $15.8(0.50-453.0)$ \\
\hline$P$ & 0.490 & & 0.815 & 0.124 & & 0.210 \\
\hline \multicolumn{7}{|l|}{$W T 1$ (missing $=2$ ) } \\
\hline Low $(n=279)$ & $43(1-83)$ & $237(50.2)$ & $42(48.8)$ & $172(61.6)$ & $107(38.4)$ & $13.5(0.34-376.8)$ \\
\hline High $(n=279)$ & $43(2-81)$ & $235(49.8)$ & $44(51.2)$ & $147(52.7)$ & $132(47.3)$ & $16.9(0.50-453.0)$ \\
\hline P & 0.873 & & 0.815 & 0.032 & & 0.406 \\
\hline \multicolumn{7}{|l|}{ GATA2 } \\
\hline $\operatorname{Low}(n=280)$ & $43(2-83)$ & $239(50.4)$ & $41(47.7)$ & $162(57.9)$ & $118(42.1)$ & $11.0(0.34-376.8)$ \\
\hline $\operatorname{High}(n=280)$ & $43(1-81)$ & $235(49.6)$ & $45(52.3)$ & $158(56.4)$ & $122(43.6)$ & $17.5(0.50-453.0)$ \\
\hline$P$ & 0.832 & & 0.639 & 0.733 & & 0.003 \\
\hline \multicolumn{7}{|l|}{$B A A L C$} \\
\hline $\operatorname{Low}(n=280)$ & $45(1-79)$ & $239(50.4)$ & $41(47.7)$ & $153(54.6)$ & $127(45.4)$ & $14.2(0.34-389.4)$ \\
\hline High $(n=280)$ & $41(1-83)$ & $235(49.6)$ & $45(52.3)$ & 167 (59.6) & $113(40.4)$ & $14.1(0.6-453.0)$ \\
\hline$P$ & 0.279 & & 0.639 & 0.232 & & 0.878 \\
\hline
\end{tabular}

WBC white blood cell, $n$ number

group. Figure 1 and Table 2 showed the relationship of cytogenetic abnormalities and gene expression level.

\section{Gene mutational status and gene expression level}

Out of 560 AML patients, 116 (20.7\%) patients were with FLT3-ITD/TKD mutations, 45 (8.0\%) were with NRAS mutations, 59 (10.5\%) were with C-KIT mutations, 100 (17.9\%) were with NPM1 mutations, 38 (6.8\%) were with WT1 mutations, 118 (21.1\%) were with CEBPA mutations, $62(11.1 \%)$ were with DNMT3A mutations, 79 (14.1\%) were with $I D H 1 / 2$ mutations, and 27 (4.8\%) were with $M L L$-PTD mutations, respectively. The distribution of gene mutations was accordant to previous reports. The detailed distribution of gene mutations in different cytogenetic risk group was shown in Additional file 4: Table S2.

The potential relationship of mutual co-existence and co-exclusion was observed between gene mutation group and investigated gene expression level. FLT3 mutations were associated with high MEIS1 $(\mathrm{P}<0.001)$, WT1 $(\mathrm{P}<0.001)$, and GATA2 $(\mathrm{P}=0.004)$ and lower $B A A L C(\mathrm{P}=0.007)$ expressions, respectively; while $C$ $K I T$ was associated with lower MEIS1 $(\mathrm{P}<0.001), W T 1$ $(\mathrm{P}<0.001)$ and GATA2 $(\mathrm{P}=0.008)$ and higher BAALC $(\mathrm{P}<0.001)$ expressions, respectively. At the same time, another class I mutation, NRAS was not associated with any gene expression level. The mutual exclusion of Class II mutations was also reflected in gene expression level. CEBPA was associated with lower $M E C O M$ $(\mathrm{P}<0.001)$, MEISI $(\mathrm{P}<0.001)$, SPI1 $(\mathrm{P}<0.001)$, and WT1 $(\mathrm{P}<0.001)$ expressions, respectively. NPM1 was related with lower ERG $(\mathrm{P}<0.001)$ and BAALC $(\mathrm{P}<0.001)$, but higher MEIS1 $(\mathrm{P}<0.001)$,WT1 $(\mathrm{P}<0.001)$ and GATA2 $(\mathrm{P}=0.001)$ expression levels, respectively. Other potential mutual relationship with gene expression level was also observed in epigenetic modifier gene mutations, 

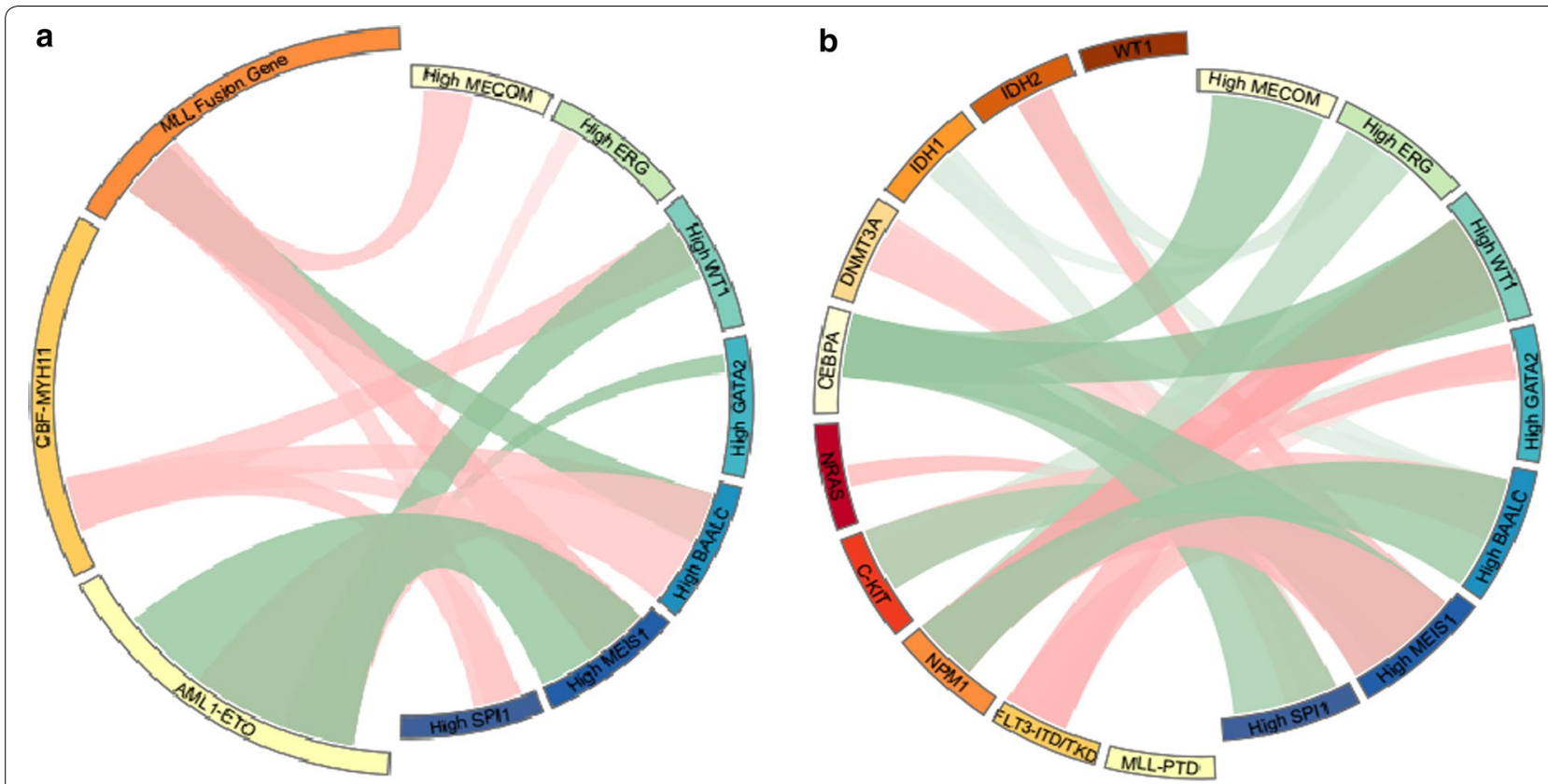

Fig. 1 Circos figure of mutual relationship between gene mutational status and investigated gene expression level. Green line indicated the negative relationship while the red line indicated positive relationship with statistical significance. a Relationship of gene fusions with gene expression level. b Relationship of gene mutations with gene expression level. The detailed numeric data were seen in Table 2 and Additional file 5: Table S3

such as DNMT3A and $I D H 1 / 2$, respectively. The detailed data were shown in Fig. 1 and Additional file 5: Table S3. Similarly, the relationship of gene mutational status and gene expression level in intermediate risk AML was shown in Additional file 6: Table S4.

\section{Treatment outcome}

\section{Response to induction therapy}

Firstly, we validated the prognostic value of known gene mutations in cytogenetic intermediate risk patients of our group and also in a separate group of young patients who received uniformed treatment. In univariate analysis, it was shown that FLT3 $(\mathrm{P}=0.036)$ and biallelic CEBPA $(\mathrm{P}=0.009)$ mutations were associated with lower and higher $C R$ rate, respectively. In different gene expression groups, higher MECOM, MEIS1 and SPI1 expression was associated with a lower CR rate $(58.0 \%$ vs. $74.4 \%, \mathrm{P}=0.001,61.2 \%$ vs. $75.8 \%, \mathrm{P}=0.002$, and $62.3 \%$ vs. $72.5 \%, \mathrm{P}=0.030$, respectively), while other factors were not associated with the induction outcome of the cytogenetic intermediate risk patients (Table 3).

A complete list of covariates that entered multivariate model was indicated in Table 4. Multivariate analysis indicated that age and cytogenetic risk remained independent prognostic factors for $\mathrm{CR}$ induction outcome, while molecular profile added more informative value in predicting the treating results, exemplified as NPM1mut $/ D N M T 3 A$-wt $(\mathrm{OR}=3.389$, 95\% CI 1.519-7.562;
$\mathrm{P}=0.003)$ and high $M E C O M$ and MEIS1 expression $(\mathrm{OR}=0.576,95 \% \mathrm{CI} 0.377-0.880 ; \mathrm{P}=0.011$, and $\mathrm{OR}=0.389,95 \%$ CI $0.251-0.603, \mathrm{P}<0.001$, respectively), respectively. A multivariate analysis was also performed in cytogenetic intermediate risk group AML patients. The results were similar in terms of gene expression level (Additional file 7: Table S5). And similar results of both univariate analysis and multivariate analysis could be observed in younger patients as well (Additional file 8: Table S6, Additional file 9: Table S7, Additional file 10: Table S8).

\section{Survival analysis}

Survival stratification was performed in the intermediate cytogenetic group, which consisted major part of AML (nearly 70\%). In our group, the median OS and DFS were observed at $17 \pm 2.3$ and $27.5 \pm 4.2$ months (24 \pm 3.1 and $30 \pm 5.0$ months for young patients), respectively (among which, the median OS and DFS of $\mathrm{CN}$-AML were $19 \pm 2.9$ and $30 \pm 4.8$ months $(26 \pm 4.8$ and $34 \pm 6.9$ months for young patients), respectively).

Firstly, survival analysis was performed according to the gene mutational status. In univariate analysis, biallelic $C E B P A$ mutations were associated with favorable OS $(\mathrm{HR}=0.329, \mathrm{P}<0.001)$ and DFS $(\mathrm{HR}=0.691, \mathrm{P}=0.002)$, respectively, as well as NPM1-mut/DNMT3A-wt (OS: $\mathrm{HR}=2.009, \mathrm{P}=0.010$; DFS: $\mathrm{HR}=2.039, \mathrm{P}=0.044$ ), which was accordant to previous study. In contrast, 
Table 2 Cytogenetic characteristics and gene expression level

\begin{tabular}{|c|c|c|c|c|c|c|}
\hline \multirow[t]{3}{*}{ Gene expression } & \multicolumn{6}{|c|}{ Cytogenetic characteristics, $n$ (\%) (failed = 15) } \\
\hline & \multicolumn{2}{|l|}{ CBF-AML } & \multicolumn{2}{|c|}{ Cytogenetic intermediate-risk } & \multicolumn{2}{|c|}{ Cytogenetic high-risk } \\
\hline & $t(8 ; 21)$ & $\operatorname{inv}(16)$ & Normal cytogenetics & Others & $11 q 23$ & Others \\
\hline MECOM & & & & & & $P<0.001$ \\
\hline Low & $34(12.5)$ & $9(3.3)$ & $181(66.8)$ & $34(12.5)$ & $5(1.8)$ & $8(3.0)$ \\
\hline High & $40(15.3)$ & $6(2.3)$ & $130(49.6)$ & $44(16.8)$ & $22(8.4)$ & $20(7.6)$ \\
\hline P & 0.364 & 0.472 & $<0.001$ & 0.165 & 0.001 & 0.015 \\
\hline MEIS1 & & & & & & $P<0.001$ \\
\hline Low & $72(26.6)$ & $1(0.4)$ & $152(56.1)$ & $30(11.1)$ & $6(2.2)$ & $10(3.7)$ \\
\hline High & $2(0.7)$ & $14(5.1)$ & $168(61.3)$ & 51 (18.6) & $21(7.7)$ & $18(6.6)$ \\
\hline P & $<0.001$ & 0.001 & 0.215 & 0.013 & 0.003 & 0.128 \\
\hline SPI1 & & & & & & $P<0.001$ \\
\hline Low & 34 (12.5) & $0(0.0)$ & $172(63.2)$ & $46(16.9)$ & $8(2.9)$ & $12(4.4)$ \\
\hline High & $40(14.7)$ & $15(5.5)$ & $148(54.2)$ & $35(12.8)$ & $19(7.0)$ & $16(5.9)$ \\
\hline P & 0.463 & $<0.001$ & 0.032 & 0.179 & 0.031 & 0.444 \\
\hline$E R G$ & & & & & & $P=0.004$ \\
\hline Low & $28(10.4)$ & $5(1.9)$ & $160(59.5)$ & $54(20.1)$ & $11(4.1)$ & $11(4.1)$ \\
\hline High & 46 (16.7) & $10(3.6)$ & $160(58.0)$ & $27(9.8)$ & $16(5.8)$ & $17(6.2)$ \\
\hline$P$ & 0.033 & 0.208 & 0.721 & 0.001 & 0.358 & 0.274 \\
\hline WT1 & & & & & & $P<0.001$ \\
\hline Low & $55(20.4)$ & $0(0.0)$ & $152(56.3)$ & $41(15.2)$ & $8(3.0)$ & $14(5.2)$ \\
\hline High & $19(7.0)$ & $15(5.5)$ & $166(60.8)$ & $40(14.7)$ & $19(7.0)$ & $14(5.1)$ \\
\hline P & $<0.001$ & $<0.001$ & 0.286 & 0.862 & 0.032 & 0.976 \\
\hline GATA2 & & & & & & $P=0.082$ \\
\hline Low & $46(17.0)$ & $10(3.7)$ & $145(53.5)$ & $44(16.2)$ & $12(4.4)$ & $14(5.2)$ \\
\hline High & $28(10.2)$ & $5(1.8)$ & $175(63.9)$ & $37(13.5)$ & $15(5.5)$ & $14(5.1)$ \\
\hline$P$ & 0.021 & 0.183 & 0.014 & 0.370 & 0.574 & 0.976 \\
\hline BAALC & & & & & & $P<0.001$ \\
\hline Low & $9(3.3)$ & $0(0.0)$ & $180(66.7)$ & $48(17.8)$ & $21(7.8)$ & $12(4.4)$ \\
\hline High & 65 (23.6) & $15(5.5)$ & $140(50.9)$ & $33(12.0)$ & $6(2.2)$ & $16(5.8)$ \\
\hline$P$ & $<0.001$ & $<0.001$ & $<0.001$ & 0.058 & 0.003 & 0.468 \\
\hline
\end{tabular}

FLT3-ITD/TKD and DNMT3A mutations were associated with poor prognosis (Additional file 11: Figure S3). Statistical significance was not observed in other gene mutation groups.

Similarly, according to gene expression level, in univariate analysis, high $M E C O M(\mathrm{HR}=1.875, \mathrm{P}<0.001$ for OS; and $\mathrm{HR}=1.558, \mathrm{P}=0.017$ for $\mathrm{DFS}$ ), high MEIS1 ( $\mathrm{HR}=1.836, \mathrm{P}<0.001$ for OS; and $\mathrm{HR}=1.550$, $\mathrm{P}=0.016$ for DFS) and high SPI1 $(\mathrm{HR}=1.402, \mathrm{P}=0.008$ for OS; and $\mathrm{HR}=1.448, \mathrm{P}=0.039$ for $\mathrm{DFS}$ ) were associated with the survival. The two parameters, MECOM and MEIS1, even had an additive effect, high MECOM and high MEIS1 group showed worst prognosis, low $M E C O M$ and low MEIS1 group presented most favorable treatment outcome, while either high MECOM or MEIS1 group was in the middle (Fig. 2i, j). WT1 could be served in further discriminating the patients with both low $M E C O M$ and MEIS1 group, the high WT1 expression was associated with poor $\mathrm{OS}(\mathrm{HR}=2.655, \mathrm{P}=0.002)$ and DFS $(\mathrm{HR}=2.889, \mathrm{P}=0.002)$.

According to above analysis and inter-relationship of gene expression level, we further stratified cytogenetic intermediate risk intermediate AML patients into 4 groups: (1) low risk: biallelic CEBPA mutations; (2) intermediate risk I: low $M E C O M$ and MEIS1 expression without biallelic CEBPA mutations or NPM1mut/DNMT3A-wt; (3) intermediate risk II: others; (4) high risk: FLT3-ITD/TKD with the absence of NPM1mut/DNMT3A-wt or DNMT3A mutations or high $M E C O M$ and MEIS1 expression (Fig. 3).

In multivariate analysis, in whole group of AML patients, age and cytogenetic risk remained independent 
Table 3 CR rate of different gene mutation and expression group in intermediate risk group

\begin{tabular}{|c|c|c|c|}
\hline Gene mutation & CR no (\%) & Gene expression & CR no (\%) \\
\hline FLT3 ITD/TKD & & MECOM & \\
\hline Mutated & $60(59.4)$ & Low & $160(74.4)$ \\
\hline Not mutated & $212(70.7)$ & High & $101(58.0)$ \\
\hline$P$ & 0.036 & $P$ & 0.001 \\
\hline NRAS & & MEISI & \\
\hline Mutated & $25(78.1)$ & Low & $138(75.8)$ \\
\hline Not mutated & $247(66.9)$ & High & $134(61.2)$ \\
\hline$P$ & 0.194 & P & 0.002 \\
\hline$C-K I T(N A=2)$ & & SPII & \\
\hline Mutated & $19(79.2)$ & Low & $158(72.5)$ \\
\hline Not mutated & $271(67.9)$ & High & $114(62.3)$ \\
\hline$P$ & 0.223 & $P$ & 0.030 \\
\hline DNMT3A & & $E R G$ & \\
\hline Mutated & $33(61.1)$ & Low & $151(70.6)$ \\
\hline Not mutated & $239(68.9)$ & High & $121(64.7)$ \\
\hline$P$ & 0.256 & P & 0.211 \\
\hline $\mathrm{IDH} 1(\mathrm{NA}=1)$ & & WT1 & \\
\hline Mutated & $23(60.5)$ & Low & $136(70.5)$ \\
\hline Not mutated & $249(68.6)$ & High & $136(66.0)$ \\
\hline$P$ & 0.311 & $P$ & 0.341 \\
\hline $\mathrm{IDH} 2$ & & GATA2 & \\
\hline Mutated & $22(62.9)$ & Low & $128(67.7)$ \\
\hline Not mutated & $250(68.3)$ & High & $144(67.9)$ \\
\hline$P$ & 0.510 & P & 0.966 \\
\hline NPM1/DNMT3A & & BAALC & \\
\hline NPM1-mut/DNMT3A-wt & $48(78.7)$ & Low & $159(69.7)$ \\
\hline Others & $224(65.9)$ & High & $113(65.3)$ \\
\hline$P$ & 0.049 & $P$ & 0.348 \\
\hline \multicolumn{4}{|l|}{ WT1 } \\
\hline Mutated & $26(76.5)$ & & \\
\hline Not mutated & $245(66.9)$ & & \\
\hline$P$ & 0.255 & & \\
\hline \multicolumn{4}{|l|}{ CEBPA } \\
\hline Biallelic mutated & $59(83.1)$ & & \\
\hline Monoallelic mutated & $21(61.8)$ & & \\
\hline Not mutated & $189(64.7)$ & & \\
\hline$P$ & 0.009 & & \\
\hline \multicolumn{4}{|l|}{ MLL-PTD } \\
\hline Mutated & $14(60.9)$ & & \\
\hline Not mutated & $258(68.3)$ & & \\
\hline P & 0.462 & & \\
\hline
\end{tabular}

prognostic factors. In terms of molecular factors, FLT3 mutations (for both OS and DFS), biallelic CEBPA mutations (for OS), NPM1-mut/DNMT3A-wt (for OS), as well as high $M E C O M$ (both for OS and DFS) and high MEIS1 (for OS) expressions were independent prognostic factors for AML (Table 4). And in intermediate risk group, similar results were also achieved (Additional file 7: Table S5). For young patients, all the above survival analysis were performed and demonstrated with the similar results (Additional file 9: Table S7, Additional file 10: Table S8; Additional file 12: Figure S4, Additional file 13: Figure S5, Additional file 14: Figure S6). 
Table 4 Multivariate analysis of prognostic value of AML

\begin{tabular}{|c|c|c|c|c|c|c|}
\hline \multirow[t]{2}{*}{ Variables } & \multicolumn{2}{|l|}{ CR } & \multicolumn{2}{|l|}{ os } & \multicolumn{2}{|l|}{ DFS } \\
\hline & OR $(95 \% \mathrm{Cl})$ & $\mathbf{P}$ & HR $(95 \% \mathrm{Cl})$ & $\mathbf{P}$ & $\mathrm{HR}(95 \% \mathrm{Cl})$ & $\mathbf{P}$ \\
\hline Age & $0.968(0.957-0.980)$ & $<0.001$ & $1.023(1.016-1.030)$ & $<0.001$ & $1.018(1.008-1.028)$ & $<0.001$ \\
\hline WBC & & NS & & NS & & NS \\
\hline Cytogenetic risk & $0.894(0.800-0.998)$ & 0.046 & $1.093(1.032-1.158)$ & 0.002 & $1.106(1.019-1.201)$ & 0.016 \\
\hline FLT3-ITD/TKD & & NS & $1.408(1.074-1.845)$ & 0.013 & $1.492(1.010-2.203)$ & 0.044 \\
\hline Biallelic CEBPA & & NS & $0.524(0.325-0.845)$ & 0.008 & & NS \\
\hline NPM1-mut/DNMT3A-wt & $3.389(1.519-7.562)$ & 0.003 & $0.523(0.355-0.770)$ & 0.001 & & NS \\
\hline MLL-PTD & & NS & & NS & & NS \\
\hline DNMT3A mutation & & NS & & NS & & NS \\
\hline High MECOM & $0.576(0.377-0.880)$ & 0.011 & $1.642(1.296-2.081)$ & $<0.001$ & $1.788(1.319-2.424)$ & $<0.001$ \\
\hline High MES/1 & $0.389(0.251-0.603)$ & $<0.001$ & $1.432(1.114-1.840)$ & 0.005 & & NS \\
\hline High SPI1 & & NS & & NS & & NS \\
\hline
\end{tabular}

NS no significance

\section{Discussion}

Cytogenetic analysis serves as a traditional tool to discriminate the prognosis of the AML [10, 43-46]. However, the shortcomings such as lack of sensitivity, labor and time cost limits the further application of this technique. Genetic mutations that escape cytogenetic detection have increasingly been discovered and these mutations may serve as potential markers to extend the prognostic parameters in AML. Numerous systemic investigations involving a series of genes have been performed in AML using first or second generation of sequencing techniques, and prognostic value has been analyzed, especially for the most common ones, such as NPM1, CEBPA, FLT3 etc. [11, 13, 47]. New generation sequencing broadens our eyes to view more deeply about these gene mutations, not only the clinical behavior and prognosis, but also the disease nature. More classes of gene mutations were named, and even used in defining the special subgroup of AML [27]. A significant progress could be observed that the diagnosis of the disease strides from a simple morphological 7 FAB subtypes to a much complicated system involving cytogenetic aberrations, gene mutations, and even gene expression levels. Nowadays, examination of gene mutations was almost routinely performed all over the world in AML field, and integrated into the daily practice in treating AML.

However, in addition to gene mutations, some gene expression levels, which are caused by the regulation of a certain gene, or even several genes, are also involved in leukemogenesis. Among them, MECOM expression was the most widely reported. It was firstly reported to be associated with a specific translocation with extreme poor prognosis, inv(3)(q21q26.2), which has lower incidence in AML $[48,49]$. Then, it was also identified to have a high expression level in AML with other cytogenetic abnormalities and even CN-AML, exemplified as in $M L L$-AF9 pediatric AML, which was associated with poor prognosis [31]; as well as in $\mathrm{M} 4 / 5$, or $M L L$ rearrangement in another Japanese series with 130 pediatric AML patients [35]. In addition to $M E C O M$, several groups use WT1 expression level to monitor the minimal residual diseases (MRD) in BM and PB in AML, whose increasing strongly indicates poor prognosis and relapse [40]. When compared with universal recognition, $M E C O M$ and WT1 over-expression are poor indicators, while clinical value of $B A A L C$ and $E R G$ are controversial. Some genes of myeloid transcriptional factors are also drawn of attraction, such as GATA2 and SPI1; especially for $M E I S 1$, which is proved to be regulated by $M L L$ mutation in previous reports [36]. Although great efforts have been made in recent decades, systemic examination of gene expression level and their cytogenetic and gene mutation background in AML is still lack. We performed this study to examine the cytogenetic abnormalities, gene mutational profile in 560 AML patients, and more importantly, a series of gene expression levels, such as MECOM, WT1, ERG, BAALC, and 3 new ones with potential value, GATA2, SPI1 and MEIS1, to try to address this question.

Firstly, we identified that certain gene expression levels were associated with the cytogenetic aberrations of the disease. In this study, MECOM expression was identified to be low in CN-AML group, but high in 11q23 aberration group. MEIS1 expression was low in $\mathrm{t}(8 ; 21)$ group, but high in the group with poor cytogenetic makers. High $B A A L C$ expression was associated with CBF-AML, but less distributed in $\mathrm{CN}$ - and 11q23 AML. Moreover, gene mutational status was also associated with the gene 


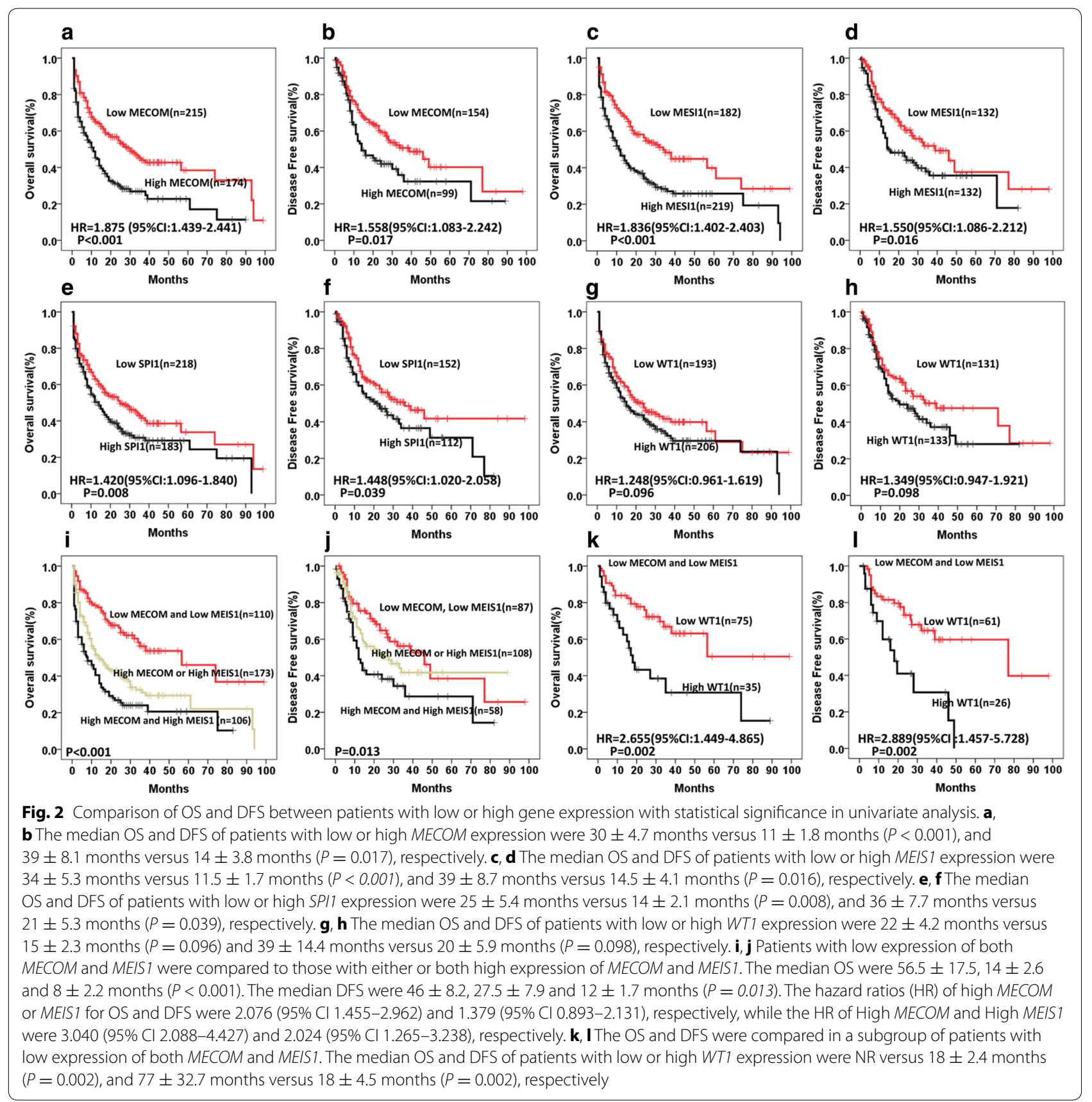

expression level. FLT3 was associated with a high MEIS1, WT1, and GATA2 and lower BAALC expression, respectively; while $C$-KIT was associated with a lower MEIS1, lower $W T 1$ and GATA2 and higher BAALC expression, respectively. And interestingly, NRAS was not associated with any gene expression level. Above results seemed to be mutually exclusive. CEBPA, as the most common mutation type in Chinese population, seemed to have favorable gene expression profile: lower MECOM $(\mathrm{P}<0.001)$, MEISI $(\mathrm{P}<0.001)$, SPI1 $(\mathrm{P}<0.001)$, and WT1
$(\mathrm{P}<0.001)$ expression, respectively. Mutant NPM1 was related with lower $E R G(\mathrm{P}<0.001), B A A L C(\mathrm{P}<0.001)$, but higher MEIS1 $(\mathrm{P}<0.001), W T 1(\mathrm{P}<0.001)$ and GATA2 $(\mathrm{P}=0.001)$, respectively. As we know, FLT3, $C-K I T, C E B P A$ and NPM1 mutations were considered to have prognostic value in predicting the prognosis of AML, and their related gene expression profile might be the reason. We believe that certain mutations elicit numerous expressional changes in other genes which are associated with leukemogenesis, leading to different 

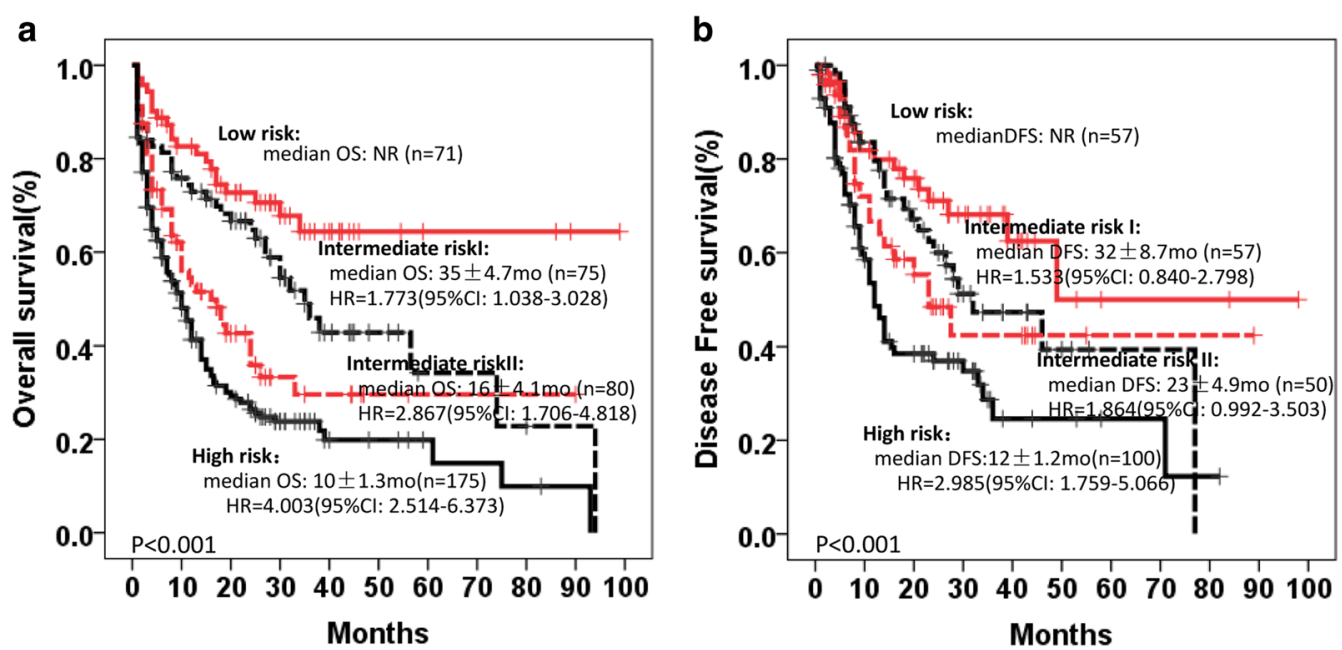

Fig. 3 Risk stratification of AML according to gene mutations and gene expression level. Low risk: biallelic CEBPA mutation; Intermediate risk I: low MECOM and MEIS1 without biallelic CEBPA mutation or NPM1-mut/DNMT3A-wt; Intermediate risk Il: others; High risk: FLT3-ITD/TKD with the absence of NPM1-mut/DNMT3A-wt or DNMT3A mutation or high MECOM and MEIS1. a OS, b DFS

clinical behaviors of the diseases. Such kind of mutual relationship with gene expression level was also observed in epigenetic modifier gene mutations.

In treatment outcome analysis, univariate analysis showed that FLT3 and higher MECOM, MEIS1 and SPI1 expressions were associated with a lower CR rate $(\mathrm{P}=0.036,0.001,0.002$ and 0.030 respectively), while CEBPA $(\mathrm{P}=0.009)$ mutations were associated with a higher $\mathrm{CR}$ rate. Higher MECOM and MEIS1 expressions remained significant in multivariate analysis, while NPM1-mut/DNMT3A-wt appeared to be an independent factor $(\mathrm{P}=0.003)$. In survival, biallelic CEBPA mutations and NPM1-mut/DNMT3A-wt were associated with favorable OS $(\mathrm{P}<0.001$ and $\mathrm{P}=0.010$, respectively $)$ and DFS ( $\mathrm{P}=0.002$ and $\mathrm{P}=0.044$, respectively), respective, while FLT3-ITD/TKD $(\mathrm{P}<0.001$ and $\mathrm{P}<0.001$, respectively) and DNMT3A ( $\mathrm{P}=0.014$ and $\mathrm{P}=0.023$, respectively) were associated with poor prognosis. As for gene expression profile, high $M E C O M(\mathrm{P}<0.001$ for $\mathrm{OS}$; and $\mathrm{P}=0.017$ for DFS, respectively) and high MEIS1 $(\mathrm{P}<0.001$ for OS; and $\mathrm{P}=0.016$ for DFS, respectively $)$ levels were associated with the survival. An additive effect could be observed when we combined the two gene expression levels together, high $M E C O M$ and high $M E I S 1$ group showed worst prognosis, low MECOM and low MEIS1 group presented most favorable treatment outcome. Furthermore, WT1 could help to separate the low risk group of low MECOM and MEIS1 group into a more refined subgroup, the high $W T 1 /$ low $M E C O M$ and MEIS1 expression was associated with relative poor OS $(\mathrm{HR}=2.655, \mathrm{P}=0.002)$ and $\mathrm{DFS}(\mathrm{HR}=2.889, \mathrm{P}=0.002)$.

Finally, we have established a new system to stratify the AML integrating cytogenetic risk, gene mutational status and gene expression profile. Through examination of traditional cytogenetic markers, gene mutations, exemplified as DNMT3A, MLL, NPM1, CEBPA, FLT3 mutations etc., and more importantly gene expression profile, especially for MECOM, MEIS1 and WT1, one could discriminate the AML patients with different clinical behaviors.

\section{Conclusions}

Gene expression aberrations are associated with the cytogenetic abnormalities and gene mutations in AML, as well as the clinical behavior of the patients. Of note, their value in predicting the prognosis of AML was demonstrated in this study. 


\section{Additional files}

Additional file 1: Figure S1. Gene expression of MECOM, ERG, WT1, GATA2, BAALC, MEIS1 and SPI1 in bone marrow (BM) of de novo AML patients was normalized to ABL expression. Each median value after a logtransformation is indicated by a horizontal line.

Additional file 2: Figure S2. Kaplan-Meier survival analysis for overall survival (OS) using different cut-offs of relative gene expression. Log-rank $P$ value was portrayed for each survival analysis.

Additional file 3: Table S1. FAB subgroups and cytogenetic abnormalities in AML patients.

Additional file 4: Table S2. The distribution of gene mutations in different cytogenetic risk groups.

Additional file 5: Table S3. The relationship of gene mutational status and gene expression level.

Additional file 6: Table S4. The relationship of gene mutational status and gene expression level (intermediate risk).

Additional file 7: Table S5. Multivariate analysis of intermediate risk group.

Additional file 8: Table S6. CR rate of different gene mutation and expression groups in intermediate risk group (young AML patients).

Additional file 9: Table S7. Multivariate analysis of prognostic value of young AML patients.

Additional file 10: Table S8. Multivariate analysis of intermediate risk group (young AML patients).

Additional file 11: Figure S3. Kaplan-Meier curves for OS and DFS according to genotypes.

Additional file 12: Figure S4. Kaplan-Meier curves of young AML patients for OS and DFS according to genotypes.

Additional file 13: Figure S5. Comparison of OS and DFS between young AML patients with low or high gene expression with statistical significance in univariate analysis.

Additional file 14: Figure S6. Risk stratification of young AML according to gene mutations and gene expression levels.

\section{Abbreviations}

AML: acute myeloid leukemia; MRD: minimal residual disease; TF: transcription factors; CN-AML: cytogenetic normal AML; CBF-AML: core binding factor AML; SIH: Shanghai Institute of Hematology; MDS: myelodysplasia syndrome BM: bone marrow; PB: peripheral blood; OS: overall survival; DFS: disease free survival.

\section{Authors' contributions}

YS and S-JC were the principal investigators who conceived the study. YS and Y-MZ coordinated and oversaw the study. Y-MZ and P-PW performed most of the experiments. P-PW completed clinical analysis. J-YH was responsible for bioinformatics investigation. P-PW, Y-SC, YH and W-YC gathered detailed clinical information for the study. Y-MZ, Y-JD, HY and T-TM participated in molecular analysis. BC and Y-MZ participated in sample bank management. YS wrote the manuscript. All authors read and approved the final manuscript.

\section{Acknowledgements}

We are grateful to Bing Chen and Shu-Min Xiong for performing the hematological morphological analysis.

\section{Competing interests}

The authors declare that they have no competing interests.

\section{Availability of data and materials}

All data generated or analysed during this study are included in this published article and its Additional files.

\section{Consent for publication}

Ethical approval by the ethic board of Ruijin hospital and consent of patients are included in the original publications.

\section{Ethics approval and consent to participate}

This study was approved by the ethic board of Ruijin hospital. All patients were given informed consent for both treatment and cryopreservation of BM and peripheral blood (PB) according to the Declaration of Helsinki.

\section{Funding}

This work was supported in part by the National Natural Science Foundation of China (81370653), National Clinical research Base construction Projects of Traditional Medicine (2012 H01) and Shanghai Municipal Education Commission-Gaofeng Clinical Medicine Grant Support (20161406). The funders had no role in study design, data collection and analysis, decision to publish, or preparation of the manuscript.

\section{Publisher's Note}

Springer Nature remains neutral with regard to jurisdictional claims in published maps and institutional affiliations.

Received: 8 June 2017 Accepted: 9 August 2017

Published online: 22 August 2017

\section{References}

1. Byrd JC, Mrozek K, Dodge RK, Carroll AJ, Edwards CG, Arthur DC, et al. Pretreatment cytogenetic abnormalities are predictive of induction success, cumulative incidence of relapse, and overall survival in adult patients with de novo acute myeloid leukemia: results from Cancer and Leukemia Group B (CALGB 8461). Blood. 2002;100(13):4325-36.

2. Mrozek K, Marcucci G, Nicolet D, Maharry KS, Becker H, Whitman SP, et al. Prognostic significance of the European leukemia net standardized system for reporting cytogenetic and molecular alterations in adults with acute myeloid leukemia. J Clin Oncol. 2012;30(36):4515-23.

3. Döhner H, Gaidzik VI. Impact of genetic features on treatment decisions in AML. ASH Educ Progr Book. 2011;2011(1):36-42.

4. Perea G, Lasa A, Aventin A, Domingo A, Villamor N, Queipo de Llano MP, et al. Prognostic value of minimal residual disease (MRD) in acute myeloid leukemia (AML) with favorable cytogenetics [t(8;21) and inv(16)]. Leukemia. 2006:20(1):87-94.

5. Guerrasio A, Pilatrino C, De Micheli D, Cilloni D, Serra A, Gottardi E, et al. Assessment of minimal residual disease (MRD) in CBFbeta/MYH11positive acute myeloid leukemias by qualitative and quantitative RT-PCR amplification of fusion transcripts. Leukemia. 2002;16(6):1176-81.

6. Yin JA, O'Brien MA, Hills RK, Daly SB, Wheatley K, Burnett AK. Minimal residual disease monitoring by quantitative RT-PCR in core binding factor AML allows risk stratification and predicts relapse: results of the United Kingdom MRC AML-15 trial. Blood. 2012;120(14):2826-35.

7. Pigazzi M, Manara E, Buldini B, Beqiri V, Bisio V, Tregnago C, et al. Minimal residual disease monitored after induction therapy by RQ-PCR can contribute to tailor treatment of patients with t(8;21) RUNX1-RUNX1T1 rearrangement. Haematologica. 2015;100(3):e99-101.

8. Cassinat B, de Botton S, Kelaidi C, Ades L, Zassadowski F, Guillemot I, et al. When can real-time quantitative RT-PCR effectively define molecular relapse in acute promyelocytic leukemia patients? (Results of the French Belgian Swiss APL Group). Leuk Res. 2009;33(9):1178-82.

9. Khan I, Altman JK, Licht JD. New strategies in acute myeloid leukemia: redefining prognostic markers to guide therapy. Clin Cancer Res Offi J Am Assoc Cancer Res. 2012;18(19):5163-71.

10. Foran JM. New prognostic markers in acute myeloid leukemia: perspective from the clinic. ASH Educ Progr Book. 2010;2010(1):47-55.

11. Schlenk RF, Dohner K, Krauter J, Frohling S, Corbacioglu A, Bullinger L, et al. Mutations and treatment outcome in cytogenetically normal acute myeloid leukemia. N Engl J Med. 2008;358(18):1909-18.

12. Shen Y, Zhu YM, Fan X, Shi JY, Wang QR, Yan XJ, et al. Gene mutation patterns and their prognostic impact in a cohort of 1185 patients with acute myeloid leukemia. Blood. 2011;118(20):5593-603. 
13. Cancer Genome Atlas Research N. Genomic and epigenomic landscapes of adult de novo acute myeloid leukemia. N Engl J Med. 2013:368(22):2059-74.

14. Fasan A, Haferlach C, Alpermann T, Jeromin S, Grossmann V, Eder C, et al. The role of different genetic subtypes of CEBPA mutated AML. Leukemia. 2014;28(4):794-803.

15. Gilliland DG, Griffin JD. The roles of FLT3 in hematopoiesis and leukemia. Blood. 2002;100(5):1532-42.

16. Su L, Gao SJ, Li W, Tan YH, Cui JW, Hu RP. NPM1, FLT3-ITD, CEBPA, and c-kit mutations in 312 Chinese patients with de novo acute myeloid leukemia. Hematology (Amsterdam, Netherlands). 2014;19(6):324-8.

17. Wakita S, Yamaguchi H, Omori I, Terada K, Ueda T, Manabe E, et al. Mutations of the epigenetics-modifying gene (DNMT3a, TET2, IDH1/2) at diagnosis may induce FLT3-ITD at relapse in de novo acute myeloid leukemia. Leukemia. 2013;27(5):1044-52.

18. Shen Y, Fu YK, Zhu YM, Lou YJ, Gu ZH, Shi JY, et al. Mutations of epigenetic modifier genes as a poor prognostic factor in acute promyelocytic leukemia under treatment with all-trans retinoic acid and arsenic trioxide. EBioMedicine. 2015;2(6):563-71.

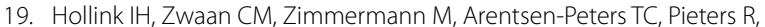
Cloos J, et al. Favorable prognostic impact of NPM1 gene mutations in childhood acute myeloid leukemia, with emphasis on cytogenetically normal AML. Leukemia. 2009;23(2):262-70.

20. Shamaa S, Laimon N, Aladle DA, Azmy E, Elghannam DM, Salem DA, et al. Prognostic implications of NPM1 mutations and FLT3 internal tandem duplications in Egyptian patients with cytogenetically normal acute myeloid leukemia. Hematology (Amsterdam, Netherlands). 2014;19(1):22-30.

21. Metzeler KH, Herold T, Rothenberg-Thurley M, Amler S, Sauerland MC, Gorlich D, et al. Spectrum and prognostic relevance of driver gene mutations in acute myeloid leukemia. Blood. 2016;128(5):686-98.

22. Krauth MT, Alpermann T, Bacher U, Eder C, Dicker F, Ulke M, et al. WT1 mutations are secondary events in AML, show varying frequencies and impact on prognosis between genetic subgroups. Leukemia. 2015;29(3):660-7.

23. Owen C, Fitzgibbon J, Paschka P. The clinical relevance of Wilms Tumour 1 (WT1) gene mutations in acute leukaemia. Hematol Oncol. 2010;28(1):13-9.

24. Krug U, Ganser A, Koeffler HP. Tumor suppressor genes in normal and malignant hematopoiesis. Oncogene. 2002;21(21):3475-95.

25. Chen YS, Wang PP, Hu Y, Zhu YM, Chen B, Huang JY, et al. Kinase-associated gene mutation pattern and clinical relevance in 205 patients with core binding factor leukemias. Blood Cancer J. 2016;6(11):e494.

26. Tao YF, Xu LX, Lu J, Cao L, Li ZH, Hu SY, et al. Metallothionein III (MT3) is a putative tumor suppressor gene that is frequently inactivated in pediatric acute myeloid leukemia by promoter hypermethylation. J Transl Med. 2014;12:182.

27. Papaemmanuil E, Gerstung M, Bullinger L, Gaidzik VI, Paschka P, Roberts ND, et al. Genomic classification and prognosis in acute myeloid leukemia. N Engl J Med. 2016;374(23):2209-21.

28. Yamazaki H, Suzuki M, Otsuki A, Shimizu R, Bresnick EH, Engel JD, et al. A remote GATA2 hematopoietic enhancer drives leukemogenesis in inv(3) (q21;q26) by activating EVI1 expression. Cancer Cell. 2014;25(4):415-27.

29. Baldus CD, Thiede C, Soucek S, Bloomfield CD, Thiel E, Ehninger G. BAALC expression and FLT3 internal tandem duplication mutations in acute myeloid leukemia patients with normal cytogenetics: prognostic implications. J Clin Oncol Off J Am Soc Clin Oncol. 2006;24(5):790-7.

30. Damm F, Heuser M, Morgan M, Wagner K, Gorlich K, Grosshennig A, et al. Integrative prognostic risk score in acute myeloid leukemia with normal karyotype. Blood. 2011;117(17):4561-8.

31. Matsuo H, Kajihara M, Tomizawa D, Watanabe T, Saito AM, Fujimoto J, et al. EVI1 overexpression is a poor prognostic factor in pediatric patients with mixed lineage leukemia-AF9 rearranged acute myeloid leukemia. Haematologica. 2014;99(11):e225-7.

32. Schwind S, Marcucci G, Maharry K, Radmacher MD, Mrozek K, Holland KB, et al. BAALC and ERG expression levels are associated with outcome and distinct gene and microRNA expression profiles in older patients with de novo cytogenetically normal acute myeloid leukemia: a Cancer and Leukemia Group B study. Blood. 2010;116(25):5660-9.

33. Nomdedeu JF, Hoyos M, Carricondo M, Bussaglia E, Estivill C, Esteve J, et al. Bone marrow WT1 levels at diagnosis, post-induction and postintensification in adult de novo AML. Leukemia. 2013;27(11):2157-64.

34. Hermkens MC, van den Heuvel-Eibrink MM, Arentsen-Peters ST, Baruchel A, Stary J, Reinhardt D, et al. The clinical relevance of BAALC and ERG expression levels in pediatric AML. Leukemia. 2013;27(3):735-7.

35. Jo A, Mitani S, Shiba N, Hayashi Y, Hara Y, Takahashi H, et al. High expression of EVI1 and MEL1 is a compelling poor prognostic marker of pediatric AML. Leukemia. 2015;29(5):1076-83.

36. Kumar AR, Li Q, Hudson WA, Chen W, Sam T, Yao Q, et al. A role for MEIS1 in MLL-fusion gene leukemia. Blood. 2009;113(8):1756-8.

37. Roychoudhury J, Clark JP, Gracia-Maldonado G, Unnisa Z, Wunderlich M, Link KA, et al. MEIS1 regulates an HLF-oxidative stress axis in MLL-fusion gene leukemia. Blood. 2015;125(16):2544-52.

38. Gonzalez Garcia JR, Meza-Espinoza JP. Use of the international system for human cytogenetic nomenclature (ISCN). Blood. 2006;108(12):3952-3.

39. Kuendgen A, Germing U. Emerging treatment strategies for acute myeloid leukemia (AML) in the elderly. Cancer Treat Rev. 2009;35(2):97-120.

40. Cilloni D, Renneville A, Hermitte F, Hills RK, Daly S, Jovanovic JV, et al. Real-time quantitative polymerase chain reaction detection of minimal residual disease by standardized WT1 assay to enhance risk stratification in acute myeloid leukemia: a European LeukemiaNet study. J Clin Oncol Offi J Am Soc Clin Oncol. 2009;27(31):5195-201.

41. Beillard E, Pallisgaard N, van der Velden VH, Bi W, Dee R, van der Schoot $\mathrm{E}$, et al. Evaluation of candidate control genes for diagnosis and residual disease detection in leukemic patients using 'real-time' quantitative reverse-transcriptase polymerase chain reaction (RQ-PCR) - a Europe against cancer program. Leukemia. 2003;17(12):2474-86.

42. Cai Q, Jeannet R, Hua W-K, Cook GJ, Zhang B, Qi J, et al. CBFß-SMMHC creates aberrant megakaryocyte-erythroid progenitors prone to leukemia initiation in mice. Blood. 2016;128(11):1503-15.

43. Flach J, Dicker F, Schnittger S, Schindela S, Kohlmann A, Haferlach T, et al. An accumulation of cytogenetic and molecular genetic events characterizes the progression from MDS to secondary AML: an analysis of 38 paired samples analyzed by cytogenetics, molecular mutation analysis and SNP microarray profiling. Leukemia. 2011;25(4):713-8.

44. Miyazaki Y, Kuriyama K, Miyawaki S, Ohtake S, Sakamaki H, Matsuo T, et al. Cytogenetic heterogeneity of acute myeloid leukaemia (AML) with trilineage dysplasia: Japan Adult Leukaemia Study Group-AML 92 study. Br J Haematol. 2003;120(1):56-62.

45. Prebet T, Boissel N, Reutenauer S, Thomas X, Delaunay J, Cahn JY, et al. Acute myeloid leukemia with translocation $(8 ; 21)$ or inversion (16) in elderly patients treated with conventional chemotherapy: a collaborative study of the French CBF-AML intergroup. J Clin Oncol Offi J Am Soc Clini Oncol. 2009;27(28):4747-53.

46. Löwenberg B. Acute myeloid leukemia: the challenge of capturing disease variety. ASH Educ Progr Book. 2008;2008(1):1-11.

47. Patel JP, Gonen M, Figueroa ME, Fernandez H, Sun Z, Racevskis J, et al. Prognostic relevance of integrated genetic profiling in acute myeloid leukemia. N Engl J Med. 2012;366(12):1079-89.

48. Levy ER, Parganas E, Morishita K, Fichelson S, James L, Oscier D, et al. DNA rearrangements proximal to the EVI1 locus associated with the 3q21q26 syndrome. Blood. 1994;83(5):1348-54.

49. Morishita K, Parganas E, Bartholomew C, Sacchi N, Valentine MB, Raimondi SC, et al. The human Evi-1 gene is located on chromosome $3 q 24-q 28$ but is not rearranged in three cases of acute nonlymphocytic leukemias containing t(3;5)(q25;q34) translocations. Oncog Res. 1990;5(3):221-31. 